\title{
同步后分析的端粒酶分周期检测新技术
}

吴霞 ${ }^{1}$, 郭玥 $^{1}$, 戴俊 ${ }^{2}$, 夏帆 $^{1}$, 娄筱叮 ${ }^{1 *}$

1. 中国地质大学材料与化学学院, 生物地质与环境地质国家重点实验室, 纳米矿物材料及应用教育部工程研究中心, 武汉 430078;

2. 华中科技大学同济医学院附属同济医院妇产科, 武汉 430030

* 联系人, E-mail: louxiaoding@cug.edu.cn

\section{A new technique for telomerase detection based on post-synchronous analysis}

\author{
Xia $\mathrm{Wu}^{1}$, Yue Guo ${ }^{1}$, Jun Dai ${ }^{2}$, Fan Xia ${ }^{1} \&$ Xiaoding Lou ${ }^{1 *}$ \\ ${ }^{1}$ State Key Laboratory of Biogeology and Environmental Geology, Engineering Research Center of Nano-Geomaterials of Ministry of Education, Fa- \\ culty of Materials Science and Chemistry, China University of Geosciences, Wuhan 430078, China; \\ ${ }^{2}$ Department of Obstetrics and Gynecology, Tongji Hospital, Tongji Medical College, Huazhong University of Science and Technology, Wuhan 430030, \\ China \\ * Corresponding author, E-mail: louxiaoding@cug.edu.cn
}

doi: 10.1360/TB-2021-0366

世界卫生组织于 2021 年2月发布的《2020年世界癌症报 告》显示, 在未来的 20 年中, 全世界的癌症病例数可能会增 加 $60 \%$. 全球每年有 $1 / 6$ 的人死于癌症, 癌症负担在增加 ${ }^{[1]}$. 根 据世界卫生组织的权威性结论, 癌症如果能在早期被发现, 患者的治愈率可达 $80 \%$. 然而, 许多癌症很难在早期查出, 一 般等到中、晚期的时候, 往往错过了最佳治疗时机. 因此, 对 肿瘤细胞的准确判断及对癌症早期的准确篮查意义重大. 端 粒酶是一种核糖核蛋白复合体，由核糖核酸模板、逆转录酶 催化组分(TERT蛋白)和其他相关蛋白组成 ${ }^{[2]}$. 在正常人体组 织中, 端粒酶的活性被抑制, 但在约 $85 \%$ 的癌细胞中, 端粒酶 被重新激活, 使肿瘤细胞能够在细胞分裂中维持端粒长度, 实现永生化 ${ }^{[3,4]}$. 因此, 端粒酶可以作为一种重要的肿瘤标志 物 $^{[5]}$. 准确检测细胞中的端粒酶活性在肿瘤疾病的诊断和预 后判断中显得尤为重要.

尽管目前报道的端粒酶活性检测方法有很多，比如化学 反应发光法、电化学方法、苂光分析方法、比色法等, 但这 些方法对细胞的前处理均是基于细胞整体非同步化水平上 进行的 ${ }^{[6-9]}$. 一个随机增殖细胞群体中存在着不同周期时相 (G1期， S期，G2期和M期)的细胞，这些细胞的生化代谢方式 各具特点, 活性物质的表达会随细胞周期呈规律性的波动. 这意味着, 这些基于混合周期时相细胞的活性靶标物质检测 方法将难以排除因周期波动带来的误差. 为了探究不同细胞 周期细胞的端粒酶活性水平差异, 我们利用通过人为诱导的 细胞同步化方法，使细胞处于同一细胞周期时相. 基于前期
本课题组 ${ }^{[10 ~ 13]}$ 对端粒酶及mRNA等肿瘤标志物检测的工作 基础, 以共价键的方式将核酸单元和聚集单元结合，建立了 基于聚集诱导发光单元(AIEgens)的上下游联动检测系统, 建 立了同步后分析的技术解析细胞周期中各个时相 TERT mRNA 和端粒酶活性的动态过程 ${ }^{[14]}$, 证实了细胞内端粒酶活 性随周期变化带来的干扰. 因此, 在对细胞活性物质进行检 测时, 必须将细胞周期的影响考虑进去, 相关研究成果发表 在National Science Review ${ }^{[14]}$.

在肿瘤的诊断过程中, 端粒酶活性随细胞周期呈规律性 波动，基于混合周期时相细胞进行端粒酶检测时，难以排除 因细胞周期波动带来的误差，有可能影响对肿瘤细胞的识别 和判断. 为了克服细胞周期异质性对端粒酶活性检测结果的 影响, 我们通过人为诱导HeLa增殖细胞群体同步化后得到不 同细胞周期的细胞，再利用PyTPA-DNA及Silole-R两种聚集 诱导发光探针对四个周期细胞中的TERT mRNA含量和端粒 酶活性进行苂光定量分析. 结果发现，通过细胞同步化后， PyTPA-DNA在G0/G1期的荧光较弱, 随着G1/S、S 期的推移, 苂光强度逐渐增大, 于 $\mathrm{S}$ 期达到最大. 然而, 在同样的条件下, PyTPA-DNA 在 $\mathrm{G} 2 / \mathrm{M}$ 期中的苂光强度则是四个周期中最小 的. 通过利用Silole-R检测四个周期下细胞的端粒酶活性，也 得出了相同的趋势. 除此之外, 我们通过传统的端粒重复扩 增方法(TRAP)也证实了端粒酶活性随周期变化的结果. 在这 里，两种聚集诱导发光分子组成的上下游联动检测信号变化 规律一致, 即说明细胞周期对逆转录蛋白TERT的mRNA表达 

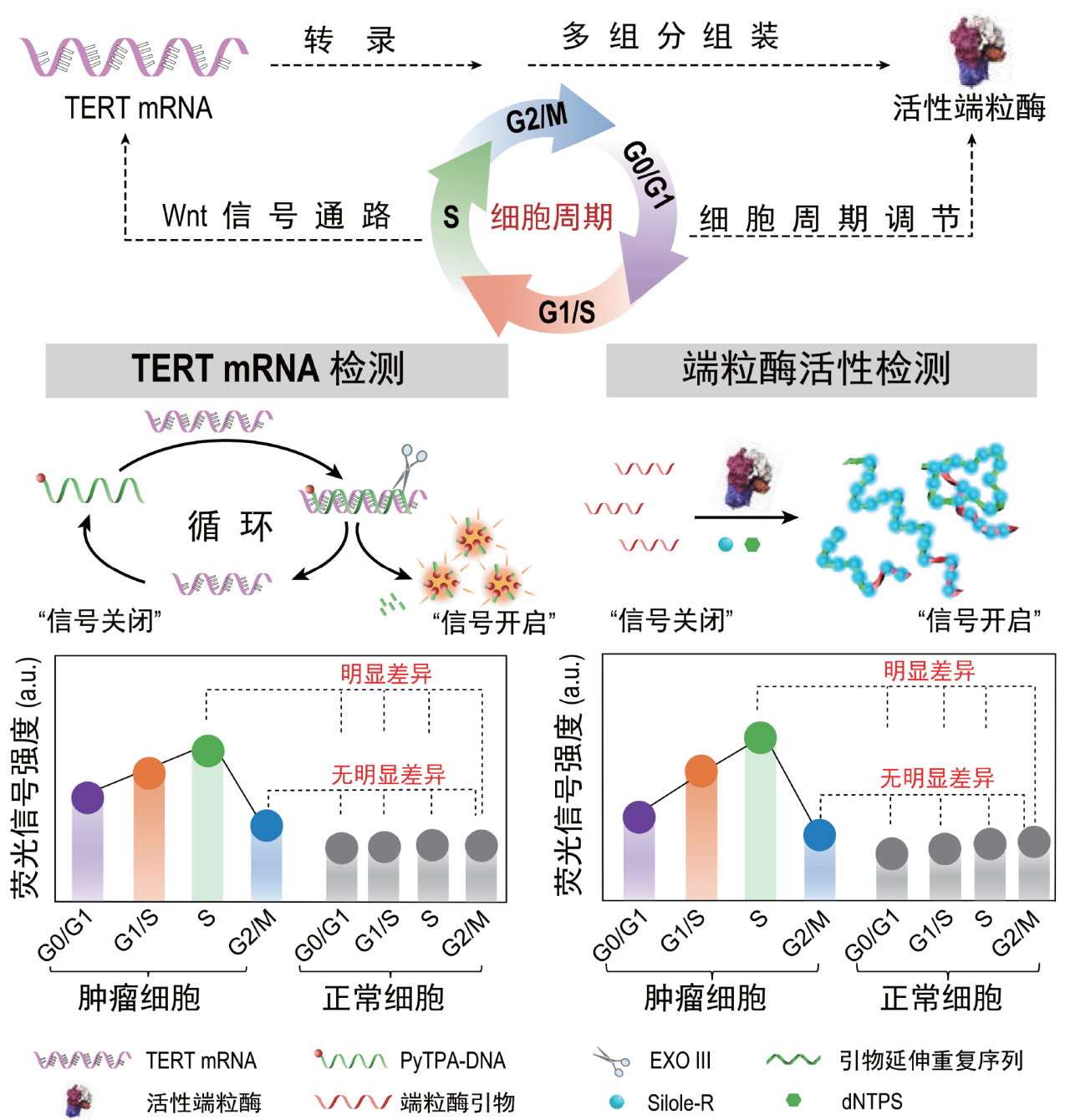

\section{端粒酶活性检测}

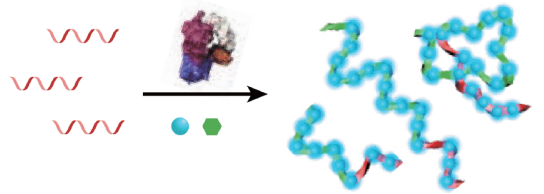

“信号关闭”

“信号开启”

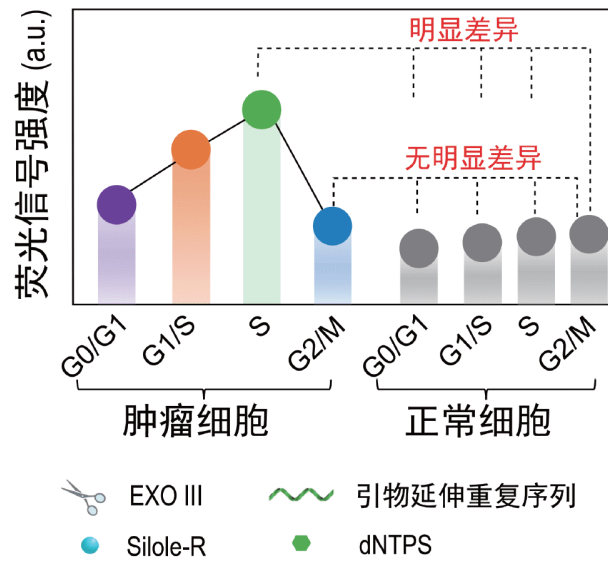

图 1 (网络版彩色)对不同周期细胞中TERT mRNA表达水平及端粒酶活性的聚集诱导发光检测系统 ${ }^{[14]}$

Figure 1 (Color online) AIEgen-based fluorescence detecting system for analysis of TERT mRNA and telomerase activity in different phases of cell cycle $^{[14]}$

及端粒酶活性均造成影响. 此外, 我们还将不同细胞周期肿 瘤细胞的TERT mRNA表达量和端粒酶活性与正常细胞进行 了对比. 结果显示, 处于 $\mathrm{G} 0 / \mathrm{G} 1 、 \mathrm{G} 1 / \mathrm{S} 、 \mathrm{~S}$ 周期的 $\mathrm{HeLa}$ 细胞中 的TERT mRNA水平和端粒酶活性均高于正常细胞; 而处于 G2/M期的Hela细胞中的TERT mRNA水平和端粒酶活性水平 与正常细胞基本相同. 这些结果都说明了在使用TERT mRNA和端粒酶活性作为肿瘤病变的早期诊断依据时应考虑 细胞周期的影响.

综上, 细胞周期对逆转录蛋白TERT mRNA及端粒酶活 性均造成影响, 两组信号变化规律一致, 从 $\mathrm{G} 0 / \mathrm{G} 1 、 \mathrm{G} 1 / \mathrm{S}$ 期逐
渐升高到 $S$ 期, G2/M期则降到最低点. 传统端粒酶检测方法 都是在细胞整体水平上进行的，忽略了周期中的异质性，可 能影响结果的准确性. 这些结果提示我们, 对肿瘤标志物进 行分周期检测是必要的. 我们通过开发周期同步后分析技术 的上下游联动检测系统实现了对TERT mRNA以及端粒酶活 性的体外及体内高精度、高灵敏度检测，为精准细胞分析提 供了一个新的切人点, 例如, 若不区分细胞周期或处于 $\mathrm{G} 2 / \mathrm{M}$ 期，则难以区分癌症细胞和正常细胞，极有可能导致漏检. 这为进一步推进癌症的早期精准诊断和治疗提供了新的 思路.

\section{推荐阅读文献}

1 Zou X L, Jia M M, Wang X, et al. Interpretation of the World Cancer Report 2020 (in Chinese). Chin J Thorac Cardiov Sur, 2021, 28: 11-18 [邹小 
农, 贾漫漫, 王金金, 等.《2020全球癌症报告》要点解读. 中国胸心血管外科临床杂志, 2021, 28: 11-18]

2 Kolquist K A, Ellisen L W, Counter C M, et al. Expression of TERT in early premalignant lesions and a subset of cells in normal tissues. Nat Genet, 1998, 19: 182-186

3 Harley C B, Kim N W, Prowse K R, et al. Telomerase, cell immortality, and cancer. Cold Spring Harb Symp Quant Biol, 1994, 59: 307-315

4 Kim N W, Piatyszek M A, Prowse K R, et al. Specific association of human telomerase activity with immortal cells and cancer. Science, 1994, 266: 2011-2015

5 Weinrich S L, Pruzan R, Ma L, et al. Reconstitution of human telomerase with the template RNA component hTR and the catalytic protein subunit hTRT. Nat Genet, 1997, 17: 498-502

6 Zhu J, Wang Y, Li B, et al. Ultra-sensitive and chemiluminescent detection of telomerase activity via handheld luminometer. Sens Actuat B Chem, 2019, 301: 127109

7 Dong P, Zhu L, Huang J, et al. Electrocatalysis of cerium metal-organic frameworks for ratiometric electrochemical detection of telomerase activity. Biosens Bioelectron, 2019, 138: 111313

8 Zhuang Y, Huang F, Xu Q, et al. Facile, fast-responsive, and photostable imaging of telomerase activity in living cells with a fluorescence turn-on manner. Anal Chem, 2016, 88: 3289-3294

9 Wang Y, Yang L, Wang Y, et al. Target-controlled in situ formation of G-quadruplex DNAzyme for a sensitive visual assay of telomerase activity. Analyst, 2019, 144: 5959-5964

10 Dai J, Wu X, Ding S Y, et al. Aggregation-induced emission photosensitizers: From molecular design to photodynamic therapy. J Med Chem, 2020, 63: 1996-2012

11 Lou X, Zhuang Y, Zuo X, et al. Real-time, quantitative lighting-up detection of telomerase in urines of bladder cancer patients by AIEgens. Anal Chem, 2015, 87: 6822-6827

12 Zhuang Y, Huang F, Xu Q, et al. Facile, fast-responsive, and photostable imaging of telomerase activity in living cells with a fluorescence turn-on manner. Anal Chem, 2016, 88: 3289-3294

13 Zhuang Y, Shang C, Lou X, et al. Construction of AIEgens-based bioprobe with two fluorescent signals for enhanced monitor of extracellular and intracellular telomerase activity. Anal Chem, 2017, 89: 2073-2079

$14 \mathrm{Wu}$ X, Wu J, Dai J, et al. Role of cell cycle progression on analyzing telomerase in cancer cells based on aggregation-induced emission luminogens. Natl Sci Rev, 2021, doi: 10.1093/nsr/nwaa306 